\title{
Neues zur pleistozänen Harzvergletscherung
}

\author{
JÜRGEN HÖVERMANN *)
}

Middle Pleistocene, Saalian glaciation, terraces, gravel, cryoturbation, paleosols, weathering, glacial tectonics, push moraine, periglacial features

\author{
Harz Mountains, Lower Saxony \\ TK 25: Nr. 4327
}

\begin{abstract}
Kurzfassung: Ein Schurf in den Böhrweg-Schottern am südwestlichen Harzrand ( $380 \mathrm{~m} \mathrm{NN}$ ) ergab überraschende Befunde hinsichtlich der Struktur der dortigen Schuttmasse. Die etwa $20 \mathrm{~m}$ mächtige Schuttmasse zeigt in den obersten Metern solifluidale und kryoturbare Strukturen, und Hinweise auf zwei ausgeprägte Naßböden, in den unteren Teilen, die im Schurf in mehr als $10 \mathrm{~m}$ Mächtigkeit aufgeschlossen waren, intensive Faltungen sowie saiger stehende und nahezu horizontale Scherflächen. Dabei ist die Verformung des Materials in den tieferen Teilen des Aufschlusses überwiegend plastisch, in den höheren, unterhalb der periglazialen Verlagerungen, überwiegend starr. Insgesamt bietet die Schuttmasse das Bild einer mehrfach durch Bodenbildungen und durch periglaziale Prozesse umgeformte Stauchmoräne.
\end{abstract}

An Grobkomponenten enthält diese Moräne ausschließlich Material von den hangaufwärts (bis $725 \mathrm{~m}$ ) anschließenden Teilen des Acker-Zuges. Das Material ist teilweise voll gerundet, teilweise eckig und zeigt neben absolut frischem Quarzit und Kieselschiefer auch völlig zermürbte, teilweise sogar zu Sand verwitterte Quarzite. In den tiefsten Teilen werden die Faltungsstrukturen besonders deutlich, da gelblich gefärbte und gebleichte Partien wechsellagern. Der Verwitterungsgrad dieser Partien deutet darauf hin, daß stark verwittertes Material mit frischem Schutt vermengt wurde.

Die Schuttmasse liegt oberhalb der Oberterrasse auf Felssockel, ist aber mit dieser durch einen Grobschuttkegel verbunden. Sie kann demnach nur oberterrassenzeitlich oder älter sein. Die Schneegrenze berechnet sich zu etwa $550 \mathrm{~m}$.

\section{[News to the Pleistocene Glaciation of the Harz Mountains]}

Abstract: Based on an exploratory excavation in the gravels of the Böhrweg at the South-West margin of the Harz ( $380 \mathrm{~m}$ over sea level), surprising results on the structure of the debris at that place were obtained. Structures of solifluction and cryoturbation in the upmost meters of a about $20 \mathrm{~m}$

*) Anschrift des Autors: Prof. Dr. J. HövermanN, Geographisches Institut Goldschmidtstraße 5, D - 3400 Göttingen. high debris have probably displaced and compressed two marked wet soil formations. Underneath intensive foldings as well as vertical and nearly horizental shear planes can be determined in exposure of more than $10 \mathrm{~m}$. Moreover in the lower parts of the exposure, the deformation of the material is mainly plastic, in the upper parts beneath the periglacial displacement mainly rigid. On the whole, the debris looks like a push moraine several times deformed by soil formation and periglacial processes.

The coarse components of this moraine are exclusively parts of the upslope (up to $725 \mathrm{~m}$ ) following ridge of the Ackerbruchberg.

Some of the material are completely rounded, angular and apart from fresh quartz and chert, there is quartzines also entirely fragmented and even partly weathered to sand. In the lowest parts a fine bedding of finer debris exists. To especially distinguish the folding structures, yellowish and etiolated parts are interbedded. The degree of weathering points out that deeply weathered material (possibly originated from the Tertiary weathering layer) and freshly sedimented debris were mixed.

The debris lies above the Uppler Terrace on a bedrock connected by a coarse talus. Consequently it can be of the age of the Upper Terrace or older than it. The snow limit is calculated at about $550 \mathrm{~m}$.

Zwischen Herzberg und Osterode liegen am südwestlichen Harzrand ausgedehnte Schotterablagerungen, die ausschließlich oder ganz überwiegend aus Harzgesteinen bestehen. Sie liegen stellenweise den $\mathrm{Ab}$ tragungsrelikten der Zechsteinformation auf, sonst unmittelbar dem Grundgebirge. Längs der Söse, von Osterode nach NW, sind sie deutlich in eine Niederterrasse (bis zu $5 \mathrm{~m}$ ü. T., lößverhüllt) und eine Oberterrasse (bis zu $80 \mathrm{~m}$ ü. T., lößfrei) gegliedert.

Alle drei Terrassen fallen nach NW um etwa $80 \mathrm{~m}$ auf 6-7 km Horizontaldistanz ab, haben also ein Gefälle von etwa 1,2\%. Die Terrassengliederung im Einzugsgebiet der Sieber, d. h. nach S und SE, ist im Prinzip 
die gleiche. Nur laufen die Schotterstränge hier fast unmittelbar vom Harzrand weg, während die Ablagerung im Sösegebiet zunächst zwischen Harzrand und Zechsteinstufe gefangen scheint.

Im Grenzbereich zwischen beiden Flußgebieten steigen diese Schotter auf den Ausläufern des AckerBruchberg-Zuges bis zu $390 \mathrm{~m}, 80 \mathrm{~m}$ über der Oberterrasse, an. In der Höhenlage von $380 \mathrm{~m}$ bis $390 \mathrm{~m}$ bilden sie eine $500 \mathrm{~m}$ lange, $250 \mathrm{~m}$ breite Hochfläche, die von HÖVERMANN (1949) der mittleren Randterrasse des Harzes zugerechnet worden ist und zeitlich dem (mittleren?) Pliozän zugeordnet wurde. Im Unterschied zu den älteren geologischen Aufnahmen, in denen alle hochgelegenen Schotter einschließlich der Böhrweg-Schotter als, ältere Schotter einheimischer Gesteine am Harzrande' (Blatt Nr. 4227 Osterode $1: 25000$, geognostisch bearbeitet von BEUSHAUSEN, BODE, ERDMANNSDÖRFFER, KAISER, KLOCKMANN, Koch, v. Koenen, SiegerT, ZeISE 1890-1905) bzw. als ,Obere Terrasse' (Blatt Nr. 4228 Riefensbeck $1: 25000$, geologisch bearbeitet durch W. SCHRIEL und STAHL, Aufnahme abgeschl. 1930) dem Diluvium zugeordnet wurden, ergab sich hieraus die Möglichkeit, daß es sich um eine korrelate Ablagerung der frühen Talbildungsphasen im Übergang der (miopliozänen?) Flächenbildung zu der durch die Aufschüttungen der Ober-, Mittel- und Niederterrasse unterbrochenen Talbildung handeln könne.

Denn wie alle Mittelgebirge Deutschlands zeigt der Harz zwischen den stockwerkartig übereinander im Sinne einer Rumpffläche angeordneten Hochflächen, deren unterste und ausgedehnteste die ,Hauptrumpffläche' HÖVERMANNs (1949) bei etwa $600 \mathrm{~m} \mathrm{NN}$ ist, und den zweifellos pleistozänen schmalsohligen bis kerbförmigen Tälern der jüngsten Talentwicklung einen Bereich, in dem Reste älterer Talböden, eingestellt auf die ,Randterrassen' des Harzes in $500 \mathrm{~m}$, $400 \mathrm{~m}$ und $300 \mathrm{~m} \mathrm{NN}$, breitsohlige und zugleich relativ steilflankige Täler erkennen lassen. Ein grundsätzlicher Unterschied zu den pleistozänen Talsohlen liegt dabei in der Breite der vorpleistozänen Talböden, die etwa das 10fache der eiszeitlichen Schotterfluren (an der gleichen Lokalität) beträgt; ein zweiter grundsätzlicher Unterschied liegt im Flußcharakter, indem sich die Flüsse der (pliozänen) Täler ausweislich der eingesenkten Talmäander als mäandrierende Flüsse zu erkennen geben, während alle pleistozänen Schotterfluren durch verwilderte Flüsse aufgeschüttet wurden (Abb. 2).

$\mathrm{Da}$ die in Frage stehenden Schotter seit Jahrzehnten nicht aufgeschlossen sind, regte die Kommission für Geomorphologie der bayerischen Akademie der Wissenschaften anläßlich einer Exkursion im Jahre 1980, die dem Studium der in Frage stehenden Probleme gewidmet war, an, durch einen Schurf nähere Er- kenntnisse über den Charakter der Böhrweg-Schotter zu gewinnen. Mit Hilfe der DFG und mit Genehmigung der zuständigen Behörden wurde im Sommer 1981 ein solcher Schurf von $22 \mathrm{~m}$ Länge, $10 \mathrm{~m}$ Breite und $11 \mathrm{~m}$ Tiefe in $390 \mathrm{~m}$ Höhe angelegt. Der Schurf verläuft WSW-ENE und liegt fast genau in der Mitte der Böhrweg-Fläche in einer Höhe von $385 \mathrm{~m}$.

\section{Das Material}

Die Analyse des Materials, das den Schotterkörper am Böhrweg kennzeichnet, konnte schon während der Aushubarbeiten durchgeführt werden. Gegenüber den früheren, nur die oberflächennahen Teile betreffenden Beobachtungen ergab sich dabei insofern eine neue Erkenntnis, als der relativ hohe Anteil an gröberen Komponenten (bis zu $40 \mathrm{~cm}$ Kantenlänge), die zugleich gut gerundet waren, sich nicht in die Teufe hin fortsetzte. Offensichtlich handelt es sich um eine Anreicherung der gröberen Komponenten an der Oberfläche im Zuge nach der Sedimentation erfolgter Umlagerungs- und Abtragungsvorgänge. Dennoch bleibt die Schuttmasse bis zur erreichten Teufe von $11 \mathrm{~m}$ kies- und blockreich. Dabei bestehen die gröberen Komponenten fast ausschließlich aus AckerBruchberg-Quarziten; in der Fraktion bis $10 \mathrm{~cm}$ dominieren Kieselschiefer. Außerdem treten vereinzelt Gangquarze und rote Sandsteine aus der AckerBruchberg-Serie auf. Relativ häufig sind TonschieferGerölle. Alle diese Komponenten sind im Einzugsbereich der Schuttmasse innerhalb der Acker-Bruchberg-Serie vertreten. Andere Harzgesteine fehlen ebenso wie sämtliche Gesteine des Harzvorlandes. Damit ist der Einzugsbereich der Ablagerung eindeutig bestimmt: Der äußerste Westteil des Acker-Bruchberg-Zuges im Bereich des Schindelgrabens zwischen $700 \mathrm{~m}$ und $400 \mathrm{~m}$. Die maximale Transportlänge beträgt $4 \mathrm{~km}$, das gesamte mögliche Einzugsgebiet umfaßt $6-7 \mathrm{qkm}$.

Scharfkantige Gesteinsbruchstücke treten nur in den obersten $1-2 \mathrm{~m}$ der Ablagerung auf. Sie sind hier, was sich insbesondere bei den Quarziten nachweisen läßt, aus der mechanischen Zerlegung ursprünglich besser, z. T. sehr weitgehend gerundeter Schotter hervorgegangen. Darunter sind alle Komponenten mindestens kantengerundet. Selbst bei Kieselschiefern finden sich Abrundungen bis zur CAILLEUX'-schen Gruppe 4; Tonschiefer sind überwiegend gut gerundet (ab Gruppe 3); die Quarzite zeigen zum Teil eine bis fast zur Kugelform gediehene Abrundung. Dabei ist die Abrundung generell um so weiter gediehen, je gröber die Gerölle sind. Das am besten gerundete Geröll, ein roter Sandstein aus der Acker-Bruchberg-Serie, zeigte bei einem Durchmesser von $2,8 \mathrm{~cm}$ die Rundung 1000. 


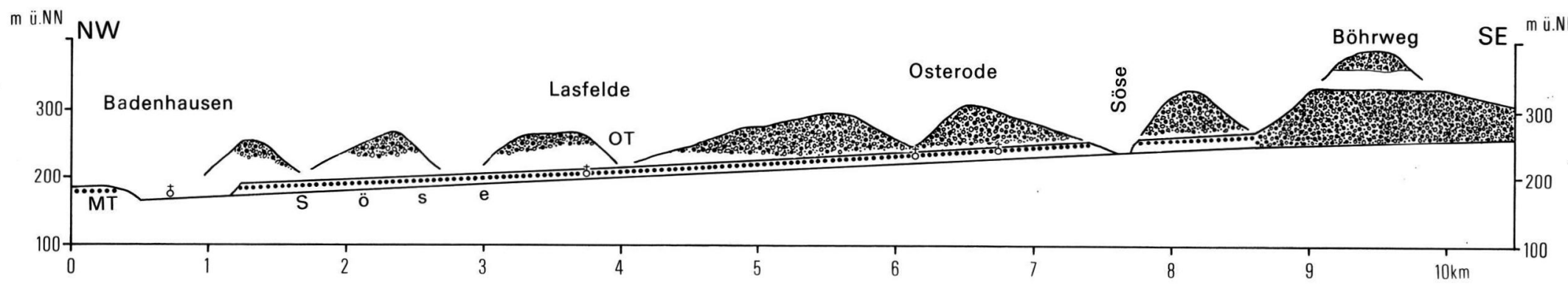

Abb. 1: Die pleistozänen Schotterterrassen zwischen Badenhausen und Osterode.

Am Böhrweg ziehen sich diese Schotter bis zu den Böhrweg-Schottern aufwärts.

Die Abbildung stellt die Auflagerungsfläche der Böhrweg-Schotter und die Fläche der OT dar.

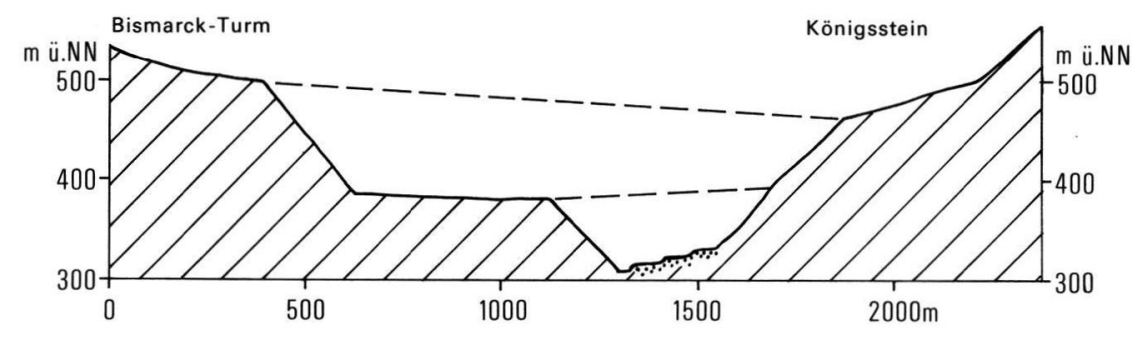

Abb. 2: „Breitterrassen” im Odertal oberhalb Bad Lauterberg. 
In der gesamten Ablagerung treten stark angewitterte Quarzite neben völlig frischen Quarziten auf. Einzelne gröbere Quarzite sind vollständig zu Sand zersetzt, obwohl die Kontur des ursprünglichen Gerölls noch zu sehen ist. Kieselschiefer erscheinen demgegenüber stets frisch. Bei den Tonschiefern läßt sich, da das Ausgangsmaterial schon sehr unterschiedlich ist, nichts über den Verwitterungsgrad aussagen. Eine differenzierte Verwitterung in situ erscheint nach den Lagerungsverhältnissen ausgeschlossen. Stark angewitterte Quarzite müssen vielmehr zusammen mit frischem Material zur Ablagerung gekommen sein. Die endgültige Zersetzung zu Sand kann allerdings in situ erfolgt sein.

Das Feinmaterial ist einesteils hell, sandig, häufig mit Lepidokrokitausscheidungen, anderenteils intensiv rötlich oder braun, meist kies- und blockreich, mit nest- oder bandartige Manganausscheidungen. In beiden Varianten des feinmaterialreichen Substrates treten Bereiche mit stark wechselnden Sand-, Schluff-, Ton- und Sequioxydanteilen auf. Dementsprechend schwanken die Farbwerte zwischen hellgrau bis rötlich-grau im Bereich der helleren, gelblich-rot (orange) bis rotbraun und braun in den dunkleren Partien.

Nach den Untersuchungen von $\mathrm{H}$. MACIY ist das Material in allen Teilen kalkfrei und, abgesehen von der Humusauflage des rezenten Bodens, humusfrei. Das Korngrößenmaximum des Feinerdeanteils liegt im Schluff-Ton-Bereich; bei einigen Proben treten auch hohe Feinsandanteile auf. An einigen Stellen sind Grundwassereinflüsse erkennbar, die auf eine möglicherweise frühweichselzeitliche Naßüberprägung des ursprünglichen Bodens hindeuten. Das Auftreten deutlich gebleichten marmorierten Materials im gesamten aufgeschlossenen Sedimentkörper weist darauf hin, daß ein ursprünglicher fossiler Boden mehrfach Naßüberprägungen ausgesetzt war und daß mehrfach Umlagerungsprozesse stattfanden.

Die sehr niedrige spezifische Kationenaustauschkapazität (um $30 \%$ ) weist mutmaßlich auf einen sehr erheblichen Kaolinanteil hin. Auffällig ist der hohe variable Ladungsanteil der $\mathrm{KAK}(\mathrm{H}: \mathrm{Al}: 80 \%$, a: $11 \%, \mathrm{Mg} 2 \%, \mathrm{~K} 14 \%$, Na $6 \%)$. Sehr hoch ist der auf Ton bezogene Eisengehalt: $12-13 \%$ bei intensiv gefärbtem Material, entsprechend ca. $18 \%$ Hämatit. Es handelt sich damit mit ziemlicher Sicherheit um eine pedogenetische Eisenanreicherung, die für ein höheres als pleistozänes Alter des Bodenmaterials spricht. Vergleichbar hohe Eisenoxydgehalte findet man bei tropischen Oxisolen. Bei ihnen liegt allerdings der Hue-Wert (Munsell) in der Regel niedriger, entsprechend der Ausfällung des Eisens als Hämatit. Die Umwandlung solcher Böden unter feucht-gemäßigten Klimabedingungen führt meist zu einer
Umwandlung des Hämatits in Goethit, dessen Farbwerte mit den hier am Boden bestimmten Farbwerten übereinstimmen. Damit ist die Vermutung begründet, daß es sich bei dem dunkleren Bodenmaterial um pleistozän-holozän umgeformte Reste eines tropischsubtropischen Oxiols handelt.

Im Gegensatz dazu spricht die starke Bleichung des sandigen Schluffs für eine Naßüberprägung des fossilen Bodens unter reduzierten Bedingungen - worauf auch die Marmorierung durch Eisenoxydhydrate hinweist - wobei der überwiegende Anteil der Eisenoxyde gelöst und lateral abgeführt wurde.

Schon während der Aushubarbeiten zeigte es sich, daß die Lagerungsverhältnisse im Bereich der Böhrweg-Schotter sehr komplizierter sind. Dank der besonders starken Regenfälle lösten sich schon nach einem Aushub von etwa $7 \mathrm{~m}$ Tiefe randliche Partien des Schurfgrabens an annähernd saiger stehenden, parallel zum Schurfgraben, also ENE-WSW verlaufenden Scherflächen $\mathrm{ab}$, an denen die verschiedenen Teile der Ablagerung in WSW-Richtung gegeneinander verschoben waren. In einem frühen Stadium der Arbeiten wurden diese Flächen als ,Drucktexturen' bezeichnet, was insofern zutrifft, als an diesen Harnischen die unterschiedlich bewegten Teile der Ablagerungsmasse in der Tat unter Druck gegeneinander bewegt wurden. Dadurch, daß die Richtung des Schurfgrabens zufällig mit der Richtung dieser Scherflächen übereinstimmte, und dank des teilweisen Einsturzes der Grabenwände konnten die Harnische praktisch über die gesamte Länge des Schurfes - $30 \mathrm{~m}$ - beobachtet werden. Meßbar waren 4 verschiedene, parallel zueinander verlaufende solche Flächen im Abstande von $30 \mathrm{~cm}$ bis $70 \mathrm{~cm}$ voneinander. Es ist anzunehmen, daß die gesamte Ablagerung auch außerhalb des Schurfes von gleichlaufenden solchen Flächen durchsetzt ist.

Senkrecht zum Verlauf des Schurfes und damit auch senkrecht zu diesen Scherflächen, d. h. mit NNW-SSE-Streichen, zeigt sich ein steil aufgerichtetes Faltensystem von großer Regelmäßigkeit, innerhalb dessen die feineren Komponenten (Schluffe, Feinsande, feinere Kiese, dünne Tonlagen) noch eine spezielle Fältelung erfahren haben. Die gröberen Komponenten stehen überwiegend vertikal, nur in den schmalen Faltenscheiteln liegen sie, dem Umbiegen der Schicht entsprechend, stellenweise horizontal. Wegen der Verschiebung der Schichten an den Scherflächen ließ sich keine der Falten quer über den Schurfgraben hinweg verfolgen. Dennoch ist das Gesamtbild des Faltenwurfes an beiden Längswänden des Aufschlusses im Prinzip gleich. Auch über die Längserstreckung, d. h. in ENE-WSW-Richtung, ließ sich eine Änderung der Lagerungsverhältnisse nicht erkennen. 


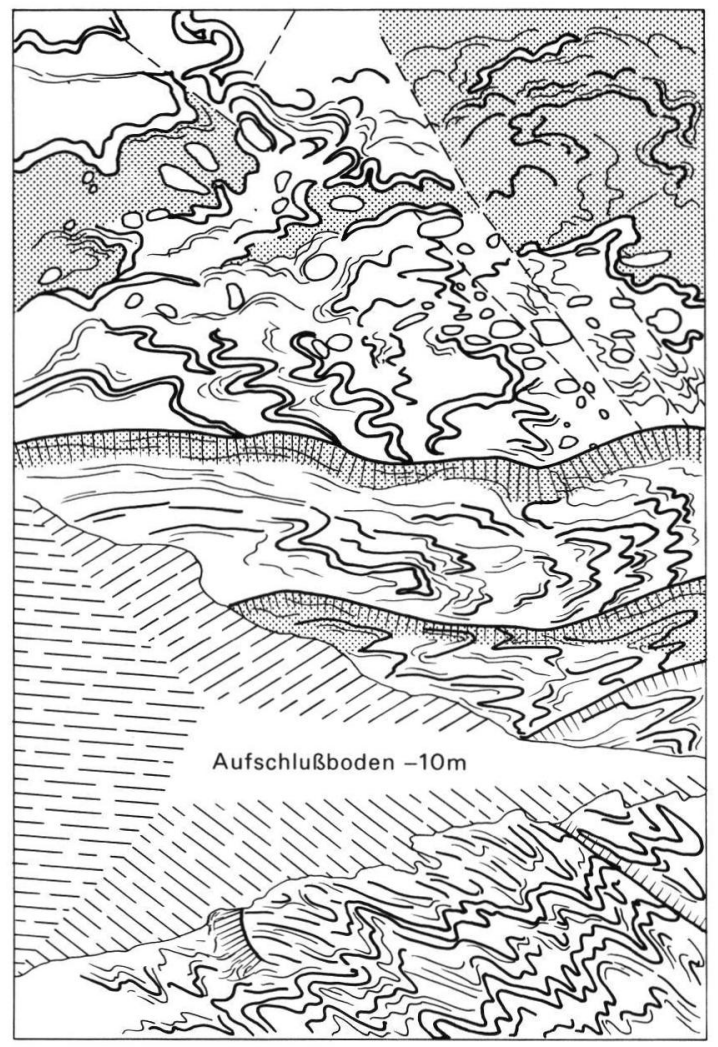

Abb. 3: Falten- und Scherstrukturen im Böhrweg-Aufschluß (nach einem Foto von H. MACIY).

Anders steht es mit der Abfolge in der Vertikalen. Während in den untersten Teilen des Aufschlusses, etwa von 6-11 m Teufe, die Falten und die Fältelungen sehr regelmäßig sind, modifiziert nur durch die Kompetenz der gefalteten Schichten, stellen sich darüber, etwa bei $4-5 \mathrm{~m}$ Teufe, stellenweise hinaufreichend bis $2 \mathrm{~m}$, zunehmend flacher liegende Scherflächen ein, die die Faltenstrukturen, überwiegend ENE oder WSW fallend, bei wahrscheinlich NNW-SSE verlaufendem Streichen, schneiden. Die Verschiebungsbeträge sind meist sehr gering und liegen im Zentimeterbereich. Trotzdem hebt sich dieser Bereich intensiverer starrer Verformung deutlich von dem liegenden Bereich ausschließlicher oder doch ganz dominierend plastischer Verformung ab (Abb. 4).

Schließlich folgt in den obersten 2-4 m wieder ein Bereich überwiegend plastischer Verformung. Doch ist hier keinerlei regelmäßiger Faltenwurf mehr erkennbar. Es handelt sich vielmehr um Materialverlagerungen unregelmäßiger Art, wie sie für Kryoturbationshorizonte und Eiskeilstrukturen charakteristisch sind. Die obersten $50-100 \mathrm{~cm}$ der Ablagerung endlich sind strukturlos und zeigen das typische Bild

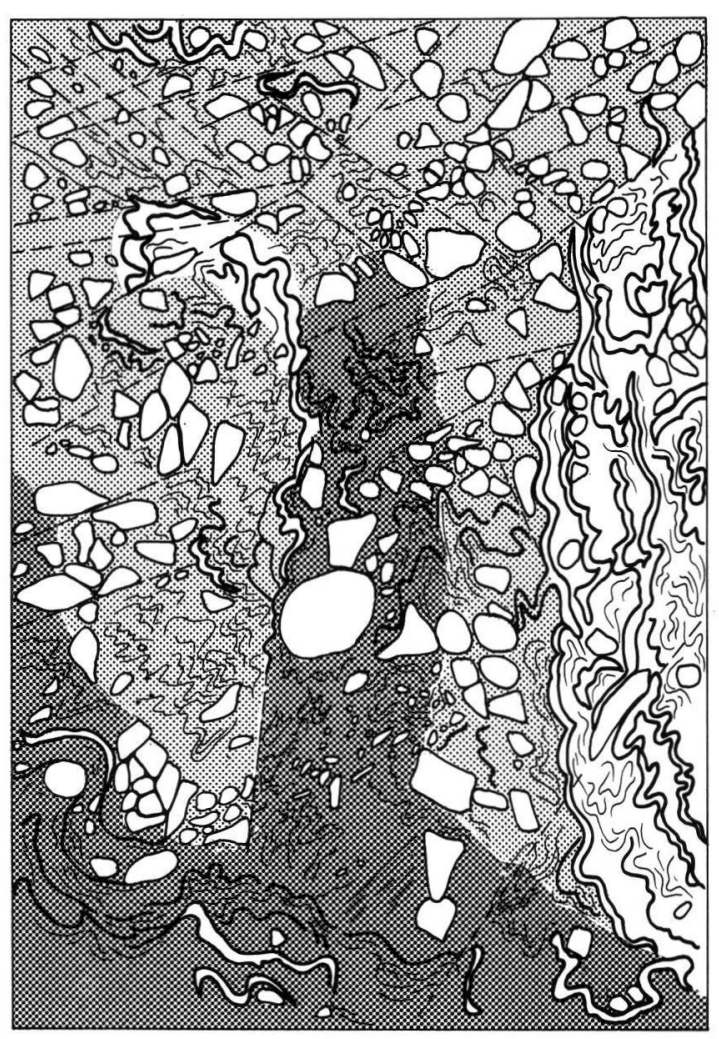

Vorwiegend hell (gebleicht)
Schwach verbraunt
Stark verbraunt
Helle Schluffbänder
Salten und Fältelungen
Schotter

Abb. 4: Falten- und Scherstrukturen in 4-5 $\mathrm{m}$ Teufe (nach einem Foto von H. MACIY).

einer Solifluktionsschuttdecke bzw. die völlige Materialdurchmengung im Bereich des Molisols eines Dauerfrostbodens (Abb. 5).

Betrachtet man die gefalteten basalen Lagen des Schotterkörpers näher, so erkennt man einen unregelmäßigen Wechsel zwischen feineren und gröberen Lagen. Tonig-schluffiges Material tritt mehrfach in dünnen Bändchen von $0,5 \mathrm{~cm}$ bis $5 \mathrm{~cm}$ Dicke auf; Kies- und Schotterlagen erreichen eine Stärke von bis zu $50 \mathrm{~cm}$. In den basalen Teilen der südexponierten Aufschlußwand ist sogar eine vielfach wiederholte Wechsellagerung von mehr schluffigem, zugleich sehr hellem, und mehr kiesigem, orangefarbigem Material zu erkennen. Stets enthalten die Schotterlagen, auch die Grobschotterlagen, lehmiges Feinmaterial. Insge- 


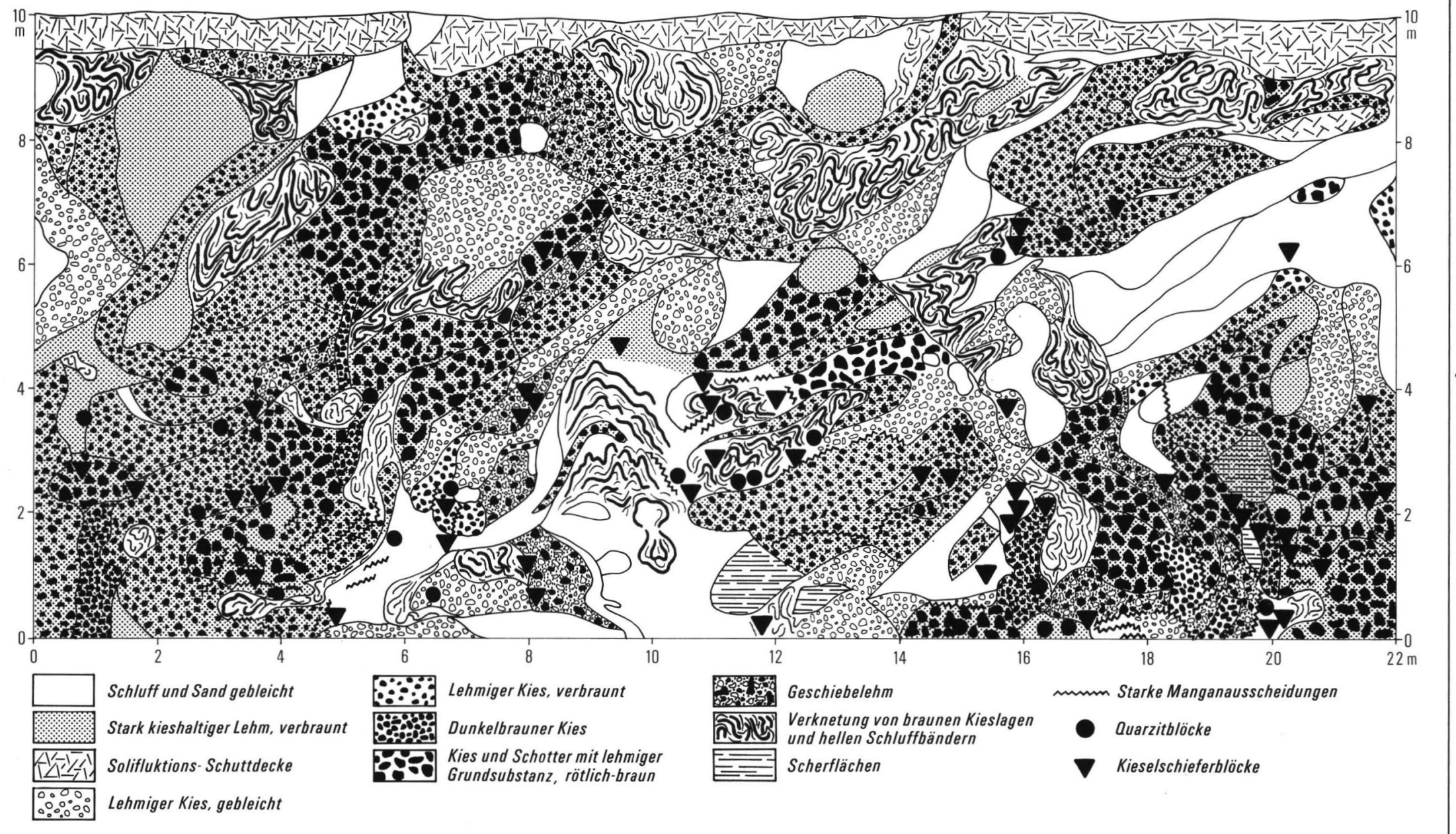

Abb. 5: Falten- und Scherstrukturen, kryoturbate Verwürgungen, Eiskeilstrukturen und Bleichungen im obersten Teil des Aufschlusses (nach einer Gitternetz-Vermessung mit $1 \mathrm{~m}$ Maschenweite von H. MACIY). 
samt handelt es sich um ein überwiegend schlecht sortiertes Schottermaterial, in dem zwar Lagen unterschiedlicher Korngröße deutlich erkennbar sind, in dem eine reinliche Materialsortierung jedoch nicht stattgefunden hat. Insofern unterscheidet sich die Ablagerung auch dann, wenn man sich die Falten ausgeglättet denkt, deutlich von pleistozänen Schottern der Mittel- und Niederterrasse, innerhalb derer die Kiese und Schotter stets gut gewaschen sind.

Dabei ist offenbar der Farbwechsel, der geringeren und höheren Gehalten an Eisen entspricht, in den tieferen Teilen des Aufschlusses keine nachträgliche Verwitterungserscheinung, sondern eine primäre Eigenschaft des Sediments. Das läßt sich daraus ableiten, daß die feineren, hellen Lagen und Bändchen ihren Charakter beibehalten, obwohl sie mehrfach an Scherflächen versetzt und auch sonst durch den Faltungsvorhang zerrissen sind. Ganz im Gegensatz dazu sind in den obersten 4-5 cm einheitliche Schotterbänke mehrfach teilweise gebleicht, teilweise intensiv braungefärbt, und zwar so, daß die höheren Partien gebleicht, die tieferen Partien durch Anlagerung von Eisenoxiden um die Grobpartikel herum gefärbt sind. Die Auffassung, daß das Sediment durch spätere Naßbleichungen überprägt worden ist, findet hierin eine Stütze.

\section{Die Genese}

Zusammen mit den Befunden über sehr unterschiedliche Zurundungsgrade innerhalb einer einheitlichen Schotterbank und mit den Befunden über das Nebeneinander von frischen und zermürbten Quarziten und die Vergesellschaft von teilweise nur kantengerundeten Kieselschiefern innerhalb einer Schotterlage läßt sich die Ablagerung vor dem Faltungsprozeß auf Sedimentationsbedingungen zurückführen, wie sie in den Randbereichen des Trockengürtels aktuell gegeben sind. Auf jeden Fall entspricht das Sediment einer fluviatilen Formung, die durch bedeutendes episodisches oder periodisches Abkommen des Flusses gekennzeichnet war, wobei sehr bedeutende Hochwässer mit dem Transport von Geröllen bis zu $40 \mathrm{~cm}$ Kantenlänge und sehr schwache Abflüsse mit der Ablagerung von Sanden und Schluffen abwechselten. Starke Abflüsse scheinen dabei dominiert zu haben.

Die Bänderung des Materials in den tieferen Partien läßt dabei darauf schließen, daß unterschiedliche Horizonte einer Verwitterungsdecke erfaßt und aufbereitet wurden, und zwar sowohl eisenreiche Anreicherungshorizonte als auch an Eisen verarmte Bleichhorizonte. Besonderer Erwähnung bedürfen fast vertikal stehende Kies- und Schotteransammlungen, die in den obersten 2-6 $\mathrm{m}$ die Ablagerung durchsetzen und die stellenweise die älteren Falten- und Scherstrukturen schneiden. Sie sind zweifellos jünger als die Faltungs- und Scherprozesse, die die Ablagerung betroffen haben, werden andererseits aber durch die Würgestrukturen im Bereich der periglazialen Überformung des Sediments, d. h. in den obersten $2-3 \mathrm{~m}$, gekappt. Es könnte sich dabei um die Füllung tieferreichender Frostspalten handeln, in denen zwar die Frostzerrung wirksam war, eine Eiskeilbildung aber nicht stattfand. Selbstverständlich könnte es sich auch um ältere Frostzerrungen handeln. Eine feinere Gliederung der (mehrfachen) periglazialen Überformung und der (mehrfachen) Naßbodenüberprägung ist nicht mit hinreichender Sicherheit gelungen. Nur bei sehr viel gutem Willen kann man in der Skizze zwei unterschiedliche Bleichzonen erkennen, die beide durch jeweils nachfolgende Solifluktions- und Kryoturbationsprozesse gestört sind. Träfe diese Interpretation des Aufschlusses zu, so waren eine schwächere (Eem bis Würm?) und eine ungleich stärkere (Treene?) Bleichung zu unterscheiden.

Deutlich wird auf jeden Fall, daß innerhalb des mit Hilfe eines $1 \mathrm{~m}$-Gitternetzes durch $\mathrm{H}$. MACIY aufgenommenen mittleren Teiles des Aufschlusses eine Auffüllung stattgefunden hat, deren Material überwiegend von WSW her kam. Das entspricht insofern den gegenwärtigen Verhältnissen, als das Gelände vom Schurf aus nach WSW auch heute leicht ansteigt. Diese Höhenunterschiede müssen vor der (mehrfachen) periglazialen Ausgleichung des Reliefs deutlich größer gewesen sein als heute. Man hat sich demzufolge am Außenrand der Ablagerung, zugleich der dem Harz abgewandten Seite, einen Wall vorzustellen, der mindestens einige Meter, vielleicht sogar mehr als $10 \mathrm{~m}$ hoch war.

Die Faltungs- und Scherprozesse, die der Ablagerung des Sedimentkörpers folgten, und die der periglazialen Überformung der obersten Partien des gestauchten und gefalteten Sediments vorausgingen, die also von der (wahrscheinlich doppelten) Bleichung und der (wahrscheinlich ebenfalls doppelten) periglazialen Verlagerung im Bereich der obersten Meter vor sich gegangen sind, stellen eine unerwartete Komplikation dar. Bei unbefangener Betrachtung würde man den aufgeschlossenen Komplex als Stauchmoräne in typischer Ausprägung anzusprechen geneigt sein. Auf diese Weise ließe sich auch die dominierend plastische Verformung in den untersten Teilen des Aufschlusses und die zusätzliche starre Verformung in den mittleren Partien dadurch erklären, daß der stauchende Gletscher über einen Dauerfrostboden hinweg vorrückte, in dessen Bereich starr verformt wurde, und darüberhinaus auch noch nicht gefrorene Teile des Sediments erfaßte, die fast ausschließlich plastisch verformt wurden. 
Die spezielle Situation im Bereich des Harzes jedoch, in dem die Indikatoren für vorzeitliche Vergletscherungen stets auf besondere (grundsätzliche) Skepsis stoßen, macht es nötig, auch andere Prozesse und Prozeßkombinationen in Erwägung zu ziehen. Als solche kommen, da die Masse nach ihrer Ablagerung als schlecht sortiertes fluviatiles Sediment gefaltet und gestaucht worden ist, nur Massenselbstbewegungen infrage. Da der Felsuntergrund, dem die Ablagerung aufliegt, im Talhang der Kleinen Steinau auf $600 \mathrm{~m}$ Länge aufgeschlossen ist und hier von $390 \mathrm{~m}$ auf $360 \mathrm{~m}$ fällt, steht ein Basisgefälle von $30 \mathrm{~m}$ auf $600 \mathrm{~m}$ $=5 \%$ zur Verfügung. Es scheint mir ausgeschlossen, $\mathrm{da} ß$ das tonarme und grobmaterialreiche Sediment unter solchen Umständen in Bewegung geraten kann, es sei denn, daß es in wasserübersättigtem Zustande sich befunden habe. So regelmäßige Faltungen, wie sie im basalen Bereich zustandegekommen sind, und so zahlreiche und engständige Scherflächen, wie sie die mittleren Teile des Sediments auszeichnen, sind mit einem solchen angenommenen Prozeß jedoch nicht in Einklang zu bringen.

Man könnte daran denken, daß im Bereich eines Dauerfrostbodens sich an der Untergrenze der Ablagerung (oder in ihren basalen Teilen) eine Eislinse gebildet habe, auf der das ganze Schotterpaket abwärts geglitten ist. Gesetz den Fall, daß die Basisfläche im Widerspruch zu den Beobachtungen im südsüdwestlichen Teil der Böhrweg-Fläche nicht vom Harz weg, sondern gegen diesen hin (nach ENE) geneigt sei (was als Möglichkeit nicht auszuschließen ist), würde die gleitende Masse auf ein Hindernis stoßen und könnte einem gewissen Zusammenschub und wohl auch Scherprozessen der beobachteten Art unterliegen. Selbstverständlich setzt diese Annahme voraus, daß das Tal der Kl. Steinau und auch das Tal der Apenke, die die Böhrweg-Fläche im SE und im NW flankieren, damals noch nicht so weit eingetieft waren, daß sie eine Drainage bewirken konnten.

Wir konzentrieren uns zunächst auf die Frage nach der Aussage der Böhrweg-Schotter für die durch steilhängige und breitsohlige Täler mit mäandrierenden Flüssen ausgezeichnete Übergangsperiode von der (miozänen?) Flächenbildung zur Bildung der schmalsohligen oder kerbförmigen pleistozänen Täler, so besagt das Sediment, daß bei stark stoßweiser Wasserführung wechselnd Schluff, Kiese und (teilweise sehr grobe) Schotter außerordentlich stark wechselnden Rundungsgrades und sehr unterschiedlichen Verwitterungszustandes abgelagert wurden, wie sie im subtropischen Übergangsbereich des ariden Gürtels heute gebildet werden. Die Beschaffenheit des Materials läßt dabei auf die Aufbereitung einer (präexistierenden) Verwitterungsdecke tropisch-subtropischen Typs schließen.
Wenn es nicht gelingt, für die Lagerungsverhältnisse und die Materialbeschaffenheit der Böhrweg-Schotter eine andere plausible Erklärung zu finden - nach dem gegenwärtigen Stand unserer Kenntnisse ist das nicht der Fall - , so scheint der Schluß auf eine HarzVergletscherung unausweichlich, die am Südwestrand des Acker-Bruchberg-Zuges bis $380 \mathrm{~m} \mathrm{NN}$ hinabreichte. Bezogen auf den höchsten Punkt des Einzugsgebietes, den Bärengarten mit gut $720 \mathrm{~m} \mathrm{NN}$, würde, in fast genauer W-Exposition, eine Schneegrenze nicht über $540 \mathrm{~m}$ abzuleiten sein. Da heute am Brocken die Höhengrenzen in W-Exposition am tiefsten liegen - ihre höchste Lage erreichen sie in SEExposition - wäre, gleiche Verhältnisse für die Eiszeiten vorausgesetzt, mit einer Schneegrenze in SEExposition um $650 \mathrm{~m} \mathrm{zu}$ rechnen. Die mittlere Schneegrenze könnte dann um $600 \mathrm{~m}$ gelegen haben. Verglichen mit den Befunden über die Würm-Eiszeitliche Vergletscherung des Harzes erscheinen diese Werte durchaus nicht so außergewöhnlich; denn die ungleich größere Ausdehnung der Eismassen während der älteren Vereisungen in Norddeutschland wie im Alpenbereich läßt einen Höhenunterschied der Schneegrenze von $100 \mathrm{~m}$ zwischen Elster, Saale (Drenthe) und Warthe einerseits, Würm andererseits durchaus plausibel erscheinen.

Die zeitliche Einordnung ist leider noch in doppelter Hinsicht unsicher. Sieht man nämlich die kontinuierliche Schotterdecke, die sich vom Böhrweg, das Anstehende verhüllend, bis zur Oberterrasse in $320 \mathrm{~m} \mathrm{NN}$ hinabzieht, als eine Art Übergangskegel an, so gehört die Stauchung der basalen und die Ablagerung der hangenden Teile der Böhrweg-Schotter der Oberterrassenzeit an. Hält man die Decke aus Schottern zwischen Böhrweg und Oberterrasse für das Ergebnis solifluidaler Verlagerung, so liegt mit dem Böhrweg ein älterer Komplex vor, der als vor-Oberterrassen-zeitlich einzustufen ist. Die zweite Unsicherheit liegt in der Datierung der Terrassen selbst. Denn die Mittelterrasse, die im Gebiet der Weser und Leine durch MENSCHING (1950) kartiert worden ist, schneidet eben jene glazialen Bildungen des Inlandeises, die der Saale-(bzw. Drenthe-) Vereisung zugerechnet werden, wird aber ungeachtet dessen wegen der Verzahnung von Leine-Schottern mit Bildungen des Inlandeises für Saale-(Drenthe-) eiszeitlich gehalten.

Setzt man, wie es morphologisch richtig ist, die Oberterrasse der Saale- (Drenthe-Stadium) Eiszeit gleich, dementsprechend die Mittelterrasse dem Warthe-Stadium, so stellt sich für die Böhrweg-Schotter die Frage Elster oder Saale (Drenthe). Folgt man der älteren auf stratigraphischen Überlegungen beruhenden Gliederung, so wäre die Frage Elster oder älter zu diskutieren. Zu diesem Komplex wird sich durch präzise Terrassenuntersuchungen eine Lösung erarbeiten lassen. 
Das Problem der Vergletscherung des Harzes während älterer Eiszeiten wirft jedoch erneut die Frage nach Vergletscherungsspuren oder wenigstens Zeugnissen vorzeitlicher nivaler Formung im deutschen Mittelgebirgsbereich ganz allgemein auf.

\section{Schriftenverzeichnis}

Bode, A. \& ERdMANNSDÖRfFer, O. H. (1907): Geol. Karte Preußen u. benachbarte Bundesstaaten 1:25000, Lfg. 100, Erl. Bl. 4227 Osterode: 50 S.; Berlin (Preuß. Geol. L.-Anst.).

DUPHORN, K. (1969): Neue Ergebnisse der Eiszeitforschung in und am Westharz. - Z. Geomorph., NF., 13: 324-334; Berlin.

GRUPE, O. (1915): Über diluviale Gebirgsstörungen im hannoverschen Berglande und zur Frage der diluvialen Hebung des Harzes. - Jb. Preuß. Geol. L.-Anst., 36: 374-400; Berlin (1916).
HÖVERMANN, J. (1949): Morphologische Untersuchungen im Mittelharz. - Göttinger geograph. Abh., 2: 80 S.; Göttingen.

- (1950): Die diluvialen Terrassen des Oberharzes und seines Vorlandes. - Petermanns geograph. Mitt., 94, 3: 121-130; Gotha.

JoRDAN, H. (1976a): Geol. Karte Niedersachsen 1:25000, Erl. Bl. 4227: Osterode: 148 S.; Hannover (Nieders. L.-Amt Bodenforsch.).

- (1976b): Die Terrassenkiese im Sösetal am Harz. Geol. Jb., A 36: 75-101; Hannover.

Mensching, H. (1950): Schotterfluren und Talauen im Niedersächsischen Bergland. - Göttinger geograph. Abh., 4: 54 S.; Göttingen.

THIEM, W. (1972): Geomorphologie des westlichen Harzrandes und seiner Fußregion. - Jb. geograph. Ges. Hannover, Sonderh. 6: 271 S.; Hannover.

Bei der Redaktion eingegangen am 30. 5. 1986. 
